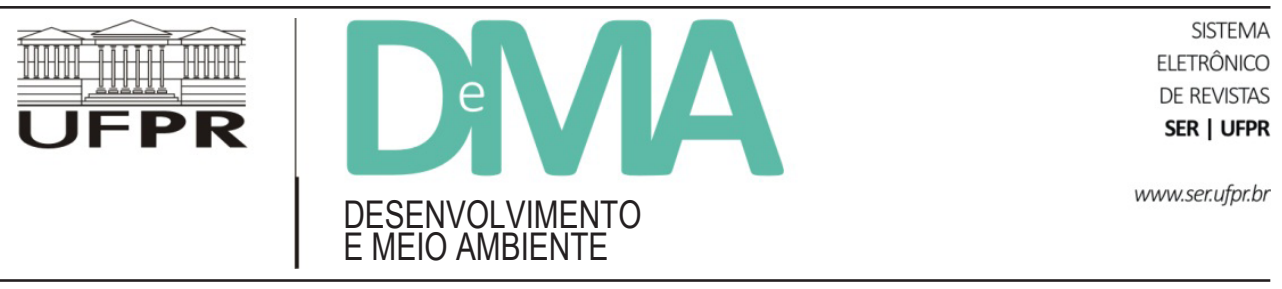

\title{
Mudanças climáticas globais e o cuidado ambiental na percepção de adolescentes: uma aproximação possível
}

\section{Global Climate Change and Environmental Care in the Perception of Adolescents: A Possible Analysis}

\author{
Hellen Chrystianne BARROS ${ }^{1 *}$, José de Queiroz PINHEIRO ${ }^{1}$ \\ ${ }^{1}$ Programa de Pós-Graduação em Psicologia (PPgPsi), Universidade Federal do Rio Grande do Norte (UFRN), Natal, RN, Brasil. \\ *E-mail de contato: hellenwbarros@hotmail.com
}

Artigo recebido em 29 de outubro de 2016, versão final aceita em 5 de fevereiro de 2017.

RESUMO: As evidências científicas da existência das mudanças climáticas globais (MCGs) são cada vez mais conhecidas, correspondendo a um problema humano-ambiental com dimensões psicológicas e sociais tanto em seu agravamento quanto em seus impactos. Este estudo objetivou investigar a percepção das MCGs por adolescentes e sua associação com práticas de cuidado ambiental autorrelatadas. Para tanto, aplicou-se um questionário, com questões abertas, a 323 adolescentes de escolas da cidade de Natal, Rio Grande do Norte. Os dados foram submetidos a análise temática de conteúdo e a análises estatísticas, a fim de investigar associações entre as variáveis. A percepção das MCGs foi considerada confusa; os adolescentes as percebem como um problema ambiental genérico e as confundem com outros problemas, como a poluição pelo acúmulo de lixo. Sobre o cuidado ambiental, $46 \%$ afirmaram praticá-lo, havendo pouca variação entre os tipos de ações mencionadas. A ação mais citada foi relativa ao controle do lixo. Os adolescentes que mencionaram praticar essas ações, assim como práticas de coleta seletiva e reciclagem, foram os que mais mencionaram causas do problema das MCGs, o que sugere que eles as percebem de maneira associada à temática do lixo. Além disso, os adolescentes que mais indicaram responsáveis pela ocorrência das MCGs foram os que relataram se engajar em ações de plantação de mudas, economia de energia elétrica e campanhas de conscientização, sugerindo a existência de associações entre a atribuição de responsabilidade pelas MCGs e o cuidado ambiental. A exploração desses conceitos busca auxiliar na promoção de medidas de educação ambiental, ampliando o escopo de ações praticadas pelos adolescentes para além das questões relativas ao lixo, pois, apesar de sua reconhecida importância, a diversidade de comportamentos também pode ser estimulada, incluindo outras ações de mitigação das MCGs em nível local.

Palavras-chave: mudanças climáticas globais; percepção; cuidado ambiental; adolescentes.

ABSTRACT: Scientific evidence of the occurrence of Global Climate Change (GCC) is increasing and becoming more widely acknowledged; it may be considered a human-environmental problem, with psychological and social 
dimensions, in regard to both its causes and consequences. This study aimed to investigate adolescents' perception of GCC in association with self-reported practices of environmental care. A questionnaire, with open questions, was applied to 323 adolescents from schools of the city of Natal, Rio Grande do Norte, Brazil. The data was subjected to thematic content and statistical analysis, in order to check for associations between variables. The perception of GCC was considered confusing; participants perceived it as a generic environmental problem, tangled with other problems, such as pollution related to the accumulation of garbage. Regarding environmental care, $46 \%$ said that they are engaged in such practices, with only minor variations of the mentioned type of actions. The most frequently cited practice was relative to waste control. Participants who reported that they engage in waste control actions, as well as selective trash collection and recycling, were the ones that mentioned causes for GCC the most, which suggests that the perception of GCC is associated with the theme of waste. In addition, the adolescents who mostly indicated people or institutions responsible for the occurrence of GCC were those who reported engaging in seedling planting, saving electricity and awareness campaigns, suggesting the existence of associations between responsibility attribution for GCC and environmental care. The exploration of such concepts aims to assist environmental education initiatives, in trying to expand the scope of adolescents' practices beyond waste matters, despite its recognized importance, as diversity of behaviors may also be stimulated in order to include other types of GCC mitigation actions at local levels.

Keywords: global climate change; perception; environmental care; adolescents.

\section{Introdução}

As mudanças climáticas globais são consideradas uma grande ameaça aos sistemas humanos e naturais e, nos últimos anos, a atenção dada ao assunto por parte da mídia e de agendas políticas e científicas internacionais tem aumentado (Clayton et al., 2015). Exemplo disso são as conferências das Nações Unidas sobre mudanças climáticas, conhecidas como COPs e realizadas no âmbito da Convenção-Quadro das Nações Unidas sobre a Mudança do Clima (CQNUMC, ou UNFCCC, do inglês United Nations Framework Convention on Climate Change), que tem como objetivos a discussão sobre as mudanças climáticas globais (MCGs), seu enfrentamento e o estabelecimento de acordos para a redução da emissão de gases de efeito estufa.

Nesse cenário, as evidências científicas sobre o papel humano no problema são cada vez mais conhecidas e consensuais (Gifford, 2008; Weber \& Stern, 2011; Clayton et al., 2015). O Painel Intergovernamental de Mudanças Climáticas, ou
Intergovernmental Panel on Climate Change (IPCC, 2014), destaca que as recentes emissões antropogênicas de gases de efeito estufa são as mais altas da história. Portanto, a ação humana deve ser diretamente envolvida também na busca de soluções e no combate às causas do problema, por meio de ações de mitigação e de medidas de adaptação em comunidades e áreas afetadas (Swim et al., 2009).

Assim, é possível conceber o aquecimento global - nome pelo qual as MCGs são popularmente conhecidas - como um problema humano-ambiental de grande complexidade, de alcance global, que envolve não apenas aspectos físicos ou bioquímicos, mas também, necessariamente, aspectos humanos: psicológicos, comportamentais, sociais e culturais (O’Neill \& Nicholson-Cole, 2009; Weber \& Stern, 2011; McDonald et al., 2015). A necessidade de estudos que levem em conta tais aspectos vem sendo apontada, mais sistematicamente, desde o início dos anos 1990, por meio de autores como Pawlik (1991) ou Stern et al. (1992), que ressaltam ainda o caráter interdisciplinar do fenômeno das MCGs (Uzzell, 2000; Stern 2011; Clayton et al., 2015). 
A psicologia é, então, convidada a contribuir com estudos a respeito das dimensões psicológicas e sociais envolvidas. Essas dimensões correspondem a esferas comportamentais, afetivas e cognitivas do funcionamento humano, que estão presentes quando se fala e se pensa em mudanças climáticas e sobre o que pode ser feito a respeito nos âmbitos local e global (Barros \& Pinheiro, 2013). As investigações desses aspectos são de grande relevância. Afinal, podem contribuir para o estímulo aos comportamentos pró-ecológicos e a ações que visem mitigar as MCGs, por exemplo. Podem, ainda, fornecer diretrizes para a implementação de projetos que visem à comunicação adequada sobre o assunto e para a aceitação de políticas públicas com vistas ao combate às MCGs (Gifford, 2008; Swim et al., 2009).

Nesse contexto, o presente estudo objetivou explorar possíveis associações entre as percepções de adolescentes relativas às MCGs e a adoção, ou não, de práticas de cuidado ambiental por eles.

Convém mencionar que já existem estudos dedicados à população adolescente e ao seu engajamento com questões ambientais (Kaiser et al., 2007; Renaud-Dubé et al., 2010; Wray-Lake et al., 2010; Ojala, 2013). Contudo, quando se trata especificamente da visão desse grupo sobre mudanças climáticas, o número de estudos existentes é ainda pequeno, tanto internacionalmente quanto no cenário nacional (Barros \& Pinheiro, 2013).

Wray-Lake et al. (2010) e Ojala (2013) ressaltam, por isso, a importância de conduzir pesquisas com adolescentes, principalmente ao considerar que serão eles os futuros cidadãos, sejam eles políticos, cientistas, empresários, professores, enfim, futuros adultos que irão lidar mais intensamente com os impactos das MCGs.

\subsection{Percepção e comunicação das mudanças climáticas globais}

Problemas ambientais globais, como as mudanças climáticas, forçam a existência de considerações sobre as escalas espaciais local e global, já que se está lidando com um problema do globo, mas que afeta as localidades de maneiras distintas e que é ali produzido (Uzzel, 2000; Clayton et al., 2015). É uma ideia comum, porém um tanto incorreta, a de que os psicólogos se preocupam apenas com os microambientes (localidades) e, assim, a investigação psicológica de questões do âmbito global seria implausível (Moser \& Uzzell, 2004). É verdade que estudar macroambientes (nível global) se constitui em um desafio heurístico (Pawlik, 2004), justamente pela abstração dessa dimensão espacial, mas é um desafio necessário diante desses problemas humano-ambientais que aí estão.

Uma das maneiras de investigar esses aspectos é, então, por meio do conceito de percepção ambiental. Esse conceito é utilizado por autores da área da psicologia ambiental referindo-se à compreensão da vivência humana nos ambientes, aludindo não apenas aos aspectos físicos destes, mas também à compreensão sobre como as pessoas entendem e interpretam seu entorno (Kuhnen \& Higuchi, 2011).

Tal concepção toma como base experiências diretas - de primeira mão - que ocorrem em ambientes locais, a que se tem acesso integral. No entanto, importa destacar que a experiência direta das MCGs é algo improvável. O sinal físico de tais alterações climáticas é extremamente baixo para ser sentido pelos humanos. As alterações são de variações baixíssimas de graus ao longo dos anos, enquanto, em um só ano, as pessoas experienciam grandes mudanças climáticas sazonais, oriundas de suas estações (Pawlik, 1991; Uzzel, 2000). 
Assim, ao considerar que a experiência direta das MCGs é improvável ou mais difícil, a percepção ambiental relativa a esse problema é construída de maneira indireta (representada, ou de segunda mão), sendo proporcionada, por exemplo, por professores em sala de aula, e/ou por sua representação em livros, jornais, internet e na mídia em geral com que se tenha contato. Tais representações, todavia, não substituem a experiência direta do mundo, do ambiente, que, no caso das MCGs, se refere à experiência dos sinais concretos desse problema nas respectivas localidades de vida dos adolescentes e em suas condições de existência (Heft \& Chawla, 2005).

Hoje, já é possível identificar presencialmente situações apontadas como consequências das MCGs para a realidade brasileira. O Painel Brasileiro de Mudanças Climáticas (PBMC, 2014), órgão nacional análogo ao IPCC, aponta, por exemplo, o avanço do nível do mar, a erosão costeira e a mudança no padrão de chuvas e secas de determinadas regiões como alguns dos principais impactos no cenário nacional. No entanto, por falta de conexão clara entre suas causas e consequências, a atribuição desses impactos às MCGs é feita com cautela (IPCC, 2014; PBMC, 2014).

Nesse sentido, a percepção das MCGs não é da ordem de fatores sensoriais, ou de psicofísica, mas, sim, da ordem da comunicação social (Pawlik, 1991). Desse modo, para haver melhor consideração do problema, a mediação comunicativa eficaz é imprescindível (Oppenheimer \& Todorov, 2006; Corner, 2012), assim como são o ensinamento e o contato com os sinais regionais das MCGs (Heft \& Chawla, 2005).

As mensagens a serem transmitidas nesse processo comunicativo devem ser, então, confiáveis, atrativas, relevantes e coerentes, e a audiência a que se destinam precisa ser motivada a agir (Clayton et al., 2015). Compreender como os adolescentes estão percebendo a questão é, então, um caminho promissor para orientar a comunicação e a educação ambiental que levem em conta tais percepções.

Os estudos realizados com populações de adultos têm revelado falta de conhecimento sobre o assunto e uma percepção confusa quando deparam com o tema (Bord et al., 1998; Dunlap, 1998; Oppenheimer \& Todorov, 2006; Sundblad et al., 2009; Gifford, 2011). Uma investigação sobre a percepção de estudantes portugueses (com idades entre 18 e 24 anos) diante das MCGs apontou falta de conhecimento claro e confusão conceitual sobre quais seriam as reais causas e os potenciais efeitos do problema (Cabecinhas et al., 2006) - resultado semelhante a que chegou estudo anterior com adolescentes brasileiros (Barros \& Pinheiro, 2013).

Isso não significa, todavia, que uma solução seria exclusivamente aumentar o conhecimento sobre dados científicos, pois o excesso desse tipo de conhecimento tem sido associado com o aumento de ceticismo (Corner, 2012). É um erro assumir que proporcionar informação descontextualizada seria suficiente para afetar decisões e comportamentos das pessoas diante das MCGs (Clayton et al., 2015). A informação é fundamental, mas outros elementos de ordem humana também são, tais como as condições contextuais dos indivíduos e como estes enxergam suas possibilidades para atuar (Gifford, 2011). Explorar eventuais aproximações entre MCGs e cuidado ambiental local parece ser uma alternativa profícua nesse sentido.

\subsection{Cuidado ambiental}

Por considerar essas possibilidades de atuação pró-ecológica na rotina das pessoas é que este estudo se interessa também pelo conceito de cuidado ambiental (Boff, 1999). 
Ao fazer uma busca simples em um dicionário on-line de português, será possível encontrar a palavra "cuidado" associada a cautela, atenção, esmero, preocupação, responsabilidade, podendo se referir à promoção de saúde, de bem-estar, à proteção de alguma coisa ou de alguém. As pessoas falam sobre ter cuidado com as crianças, ou com seus objetos pessoais, com os animais e plantas. Dessa forma, a noção de "cuidado" e esses implícitos significados são muito utilizados no dia a dia dos indivíduos, sendo, por isso, de fácil compreensão e apreensão, seja em um contexto leigo, seja em um contexto técnico-científico (Pinheiro \& Pinheiro, 2007).

Por isso, a expressão "cuidado ambiental" tem sido utilizada no sentido de proteção ecológica (Hart, 1997) em projetos e campanhas de educação ambiental, por se supor que será bem compreendida pelo público em geral (Pinheiro \& Pinheiro, 2007; Raymundo \& Kuhnen, 2010). No entanto, ainda é pouco utilizada na literatura da área de psicologia ambiental (Pinheiro \& Pinheiro, 2007). Nesse sentido, cuidado ambiental é um conceito que caminha na mesma direção de "comportamento pró-ecológico" (Corral-Verdugo, 2001). Contudo, não significa apenas um comportamento, mas um grupo de práticas cujo propósito é a proteção do meio ambiente (Pinheiro \& Pinheiro, 2007).

Este estudo não trata o cuidado ambiental como uma medida de comportamento pró-ambiental geral (Kaiser, 1998), que expressaria uma postura geral por meio da qual as pessoas se comportam e que orienta as diversas ações de proteção. Este estudo adota uma visão multidimensional do cuidado. Afinal, problemas ambientais podem significar coisas diferentes para pessoas diferentes (Gurgel \& Pinheiro, 2011) e, por isso, elas podem se engajar em certas práticas e em outras, não, dependendo do contexto e de fatores culturais. Por exemplo, uma pessoa pode economizar água, mas pode não reutilizar produtos plásticos. Importa destacar que a literatura da área assume que esses comportamentos podem ser intencionais ou não (Pato \& Campos, 2011), ou seja, ainda que o comportamento pró-ecológico pressuponha deliberação (Pinheiro \& Pinheiro, 2007; Corral-Verdugo, 2010), ele pode ocorrer por motivações diversas, tais como economia doméstica e financeira.

Nessa direção, a meta da proteção ambiental e o objeto a que esta se destina também podem ser diversos, gerando diferentes comprometimentos de acordo com interesses individuais distintos: cuidado com árvores, animais, oceanos, pessoas ou com o planeta. Esses objetos de interesse podem ir desde o nível espacial mais micro para o mais macro, da escala local até a global (Gurgel \& Pinheiro, 2011).

Tal visão multidimensional do cuidado ambiental permite, por fim, que se questione quais são as ações de cuidado ambiental praticadas pelos adolescentes e se investigue se essas ações consideram o cenário das MCGs.

\section{A proposta do estudo}

Diante da complexidade envolvida, é um tanto nebuloso o status da noção de MCGs como parte dos problemas ambientais. Nesse sentido, interessava-nos conhecer a visão de adolescentes sobre a questão, mas não conseguimos encontrar tal informação por meio do levantamento bibliográfico realizado na literatura da área no cenário nacional. Por isso, este estudo assumiu um caráter exploratório e pretendeu investigar: (a) a percepção das MCGs pelos adolescentes participantes; (b) as ações de cuidado ambiental em que esses adolescentes estão se engajando; e (c) as eventuais associações entre a percepção das MCGs pelos adolescentes e suas práticas autorrelatadas de cuidado ambiental. 


\section{Método}

\subsection{Participantes}

Participaram deste estudo 323 adolescentes, estudantes do nono ano do ensino fundamental e das primeira e segunda séries do ensino médio de duas escolas particulares e três escolas públicas da cidade de Natal, Rio Grande do Norte. Desse total, 202 (ou 63\%) eram adolescentes do sexo feminino, e 121 (ou 37\%), do sexo masculino. A média de idade foi de 15,01 anos ( $\mathrm{DP}=1,32$ ), variando de 12 a 19 anos. Os estudantes e suas escolas foram determinados por conveniência nesta investigação exploratória; tal composição não visou a qualquer propósito de representatividade populacional para além do grupo de participantes.

\subsection{Instrumentos e procedimentos}

Este estudo faz uso de uma abordagem multimetodológica (Sommer \& Sommer, 1997), estratégia que é sugerida para estudos na área de psicologia ambiental (Günther et al., 2008; Winkel et al., 2009). Os dados quantitativos e qualitativos foram coletados concomitantemente e integrados em um banco de dados, transformando os temas qualitativos em contagens e comparando estas com os dados quantitativos.

Para isso, um questionário foi aplicado coletivamente, na escola, durante o horário regular de aula, de 50 minutos, que foi disponibilizado pela direção das instituições que aceitaram participar do estudo. A participação destas, e dos adolescentes que aceitaram tomar parte na pesquisa, foi voluntária e não foram oferecidos incentivos para isso, tais como notas extras.
O questionário continha inicialmente questões relativas aos dados sociodemográficos, seguidas de uma questão para avaliar o cuidado ambiental, finalizando com uma questão aberta para investigar a percepção a respeito das MCGs, explicitadas subsequentemente.

Importa ressaltar que outros estudos também adotaram questionários como uma técnica para coletar informação a respeito das percepções sobre MCGs, ainda que de formas variadas (Cabecinhas et al., 2006; Sundblad et al., 2009). Existe na literatura da área o uso tanto de questões abertas quanto fechadas, por meio de afirmações, as quais os participantes deveriam avaliar como verdadeiras ou falsas (Dunlap, 1998; Sundblad et al., 2009).

Neste estudo, a questão aberta para registrar a percepção das MCGs foi escolhida por duas razões. Primeiro, porque nas buscas bibliográficas que realizamos não encontramos estudos anteriores sobre o tema na realidade brasileira, lidando com adolescentes. E, em situações dessa natureza, exploratória, em que não se conhece a variabilidade de respostas, questões abertas são recomendadas, pois permitem contemplar qualquer tipo de conteúdo na resposta (Günther, 2008). Além disso, em estudos-pilotos previamente realizados, foram propostas quatro perguntas abertas, gerando respostas repetitivas, evidenciando que um formato ideal poderia conter apenas uma pergunta aberta, o que foi confirmado por novo estudo-piloto.

A questão sobre MCGs recebeu um formato final específico, dentre outros testados, que implicava um caráter imaginativo, em que se solicitava ao participante que se imaginasse explicando o problema das MCGs para um amigo e, assim, se questionava o que seria falado sobre o assunto. Esse formato foi bem recebido pelos adolescentes participantes, que responderam de maneira mais completa à questão, havendo menos dúvidas. 
A questão sobre cuidado ambiental indagava ao participante se ele pratica ou já havia praticado qualquer tipo de ação que poderia ser considerada como de cuidado com o meio ambiente. As opções de resposta eram "sim" ou "não". Em caso afirmativo, era solicitado que ele fornecesse uma breve descrição da atividade praticada. Essa descrição era necessária para considerar a resposta como afirmativa, ou seja, se o participante marcasse "sim", mas não descrevesse a(s) ação(ões), a resposta era categorizada como "não". Vale ressaltar que não pedimos ao respondente qualquer definição sobre cuidado ambiental, mas solicitamos exemplos de comportamentos que ele considerasse como de cuidado. Tal estratégia permitiu o relato espontâneo do que os adolescentes entendem como cuidado. Em outros estudos que utilizaram essa mesma estratégia, não foram registradas quaisquer dificuldades de compreensão dessa noção pelo respondente ( $\mathrm{Pi}$ nheiro \& Pinheiro, 2007; Diniz \& Pinheiro, 2014).

Convém mencionar que este estudo atendeu aos requisitos éticos para pesquisas com seres humanos, tendo sido aprovado pelo Comitê de Ética da Universidade Federal do Rio Grande do Norte (UFRN).

\subsection{Análise dos dados}

As questões abertas foram transcritas para arquivo de texto e submetidas à análise temática de conteúdo (Bardin, 1977; Sommer \& Sommer, 1997; Bauer, 2002). A análise de conteúdo, técnica para descrição sistemática do conteúdo de determinado material escrito ou falado (Sommer \& Sommer, 1997), permite a aplicação simultânea de técnicas qualitativas e quantitativas, pois, a partir desse processo qualitativo de categorização, podem-se estabelecer as categorias representativas dos tópicos abordados que podem ser mensuradas e relacionadas com outras variáveis (Bauer, 2002), constituindo parte importante de um estudo de características multimetodológicas.

As categorias indicativas das percepções sobre MCGs foram estabelecidas com base na literatura da área (Dunlap, 1998; Uzzel, 2000; Cabecinhas et al., 2006; Sundblad et al., 2009; Swim et al., 2009) e nas leituras subsequentes do material. Elas foram adotadas para contemplar o discurso explícito do respondente, de modo que cada trecho de resposta representava uma categoria, não havendo sobreposição de significados entre elas. Essas categorias não eram mutuamente excludentes, ou seja, um participante poderia mencionar trechos referentes a uma ou mais categorias em sua resposta.

As categorias foram inseridas em banco de dados, juntamente com as categorias relativas às ações de cuidado ambiental autorrelatadas. Estas foram estabelecidas também com base em estudos anteriores (Corral-Verdugo, 2001; Pinheiro \& Pinheiro, 2007) e nas leituras subsequentes do material. Os dados foram submetidos a análises estatísticas a fim de investigar associações entre as variáveis do estudo. Para isso foram estabelecidas frequências e porcentagens e utilizadas provas de qui-quadrado, considerando $\mathrm{p} \leq 0,05$ como nível de significância aceitável (Tabachnick \& Fidell, 1996).

\section{Resultados e discussão}

\subsection{Como os participantes percebem as MCGs?}

Para o propósito deste artigo, esta seção descreve de maneira sumária as principais categorias e subcategorias mencionadas pelos respondentes a respeito das MCGs. Uma narrativa mais completa sobre a criação dessas categorias pode ser encontra- 
da em artigo anterior dos autores, dedicado exclusivamente a tais análises (Barros \& Pinheiro, 2013).

A Tabela 1 evidencia as frequências de ocorrência das categorias estabelecidas e suas correspondentes porcentagens em relação ao total de categorias mencionadas pelos 323 participantes e em relação ao número de participantes que as mencionaram.

TABELA 1 - Frequência absoluta e porcentagens de ocorrência de categorias estabelecidas.

\begin{tabular}{lccc}
\hline & \multicolumn{3}{c}{ Frequência de ocorrência } \\
\cline { 2 - 4 } Categorias & Absoluta & \multicolumn{2}{c}{ Percentual total de } \\
\cline { 2 - 4 } & & $\begin{array}{c}\text { Categorias } \\
(N=567)\end{array}$ & $\begin{array}{c}\text { Participantes } \\
(N=323)\end{array}$ \\
\hline Causas das MCGs & 173 & $30 \%$ & $54 \%$ \\
Mensagens de conservação & 139 & $25 \%$ & $43 \%$ \\
Atribuição de & 92 & $16 \%$ & $28,5 \%$ \\
responsabilidade & 88 & $15 \%$ & $27 \%$ \\
Consequências das MCGs & 43 & $8 \%$ & $13 \%$ \\
Situação futura & 32 & $6 \%$ & $10 \%$ \\
Soluções & & \multicolumn{2}{c}{}
\end{tabular}

As causas das MCGs foram a categoria mais mencionada pelos adolescentes (173 menções). Essa categoria foi assinalada sempre que a resposta continha explicação de causalidade, tal como exemplificado no trecho de um dos respondentes: "O aquecimento global está ocorrendo muito por causa de um grande acúmulo de lixos".

Na percepção dos participantes, as MCGs são principalmente causadas pela poluição oriunda de outros problemas ambientais, tais como do lixo depositado em locais equivocados, ou da poluição dos rios (45 menções das 173). Causas referentes à utilização de combustíveis fósseis e à emissão de gases do efeito estufa, por exemplo, foram pouco mencionadas (24/173).

As consequências das MCGs receberam menor atenção, tendo obtido 88 menções, aproximada- mente metade das indicações de causa. Sundblad et al. (2009) também evidenciaram essa tendência, sugerindo que as causas parecem ser mais comunicadas às pessoas do que as consequências e/ou mais bem percebidas e retidas pelas pessoas. Essa categoria foi assinalada quando os respondentes forneceram os efeitos, os impactos do problema, tal como observado no seguinte exemplo de resposta: “... essa destruição gera alguns problemas como o derretimento das calotas polares". Algumas respostas mencionavam mais de um tipo de consequência, como por exemplo: “... acarreta muitos problemas, como o aumento da temperatura, o aumento do nível dos oceanos, e provoca enchentes".

As consequências mais mencionadas, portanto, foram o derretimento das geleiras (41/88) e o aumento da temperatura global (30/88), sendo poucas as indicações de implicações para a realidade local; somente 12 adolescentes dos 88 que indicaram consequência mencionaram o aumento da temperatura especificamente em suas cidades.

Esses dados sobre causas e consequências são sinais de que as MCGs não estão sendo bem apreendidas pelos adolescentes participantes do estudo, pois eles não mencionaram a existência de eventos climáticos extremos como impactos do problema, e a menção ao uso de combustíveis fósseis foi inexpressiva. Embora não seja possível afirmar que tais fatos não estão sendo percebidos, surpreende que não tenham comparecido nas respostas sobre MCGs. Ainda que existam posicionamentos que discutem incertezas associadas ao problema, o esclarecimento conceitual é necessário, com vistas à apropriação do debate e ao engajamento em soluções (Clayton et al., 2015).

Houve apenas 32 menções de possíveis soluções. Estas foram assinaladas quando o respondente mencionava ações que afirmavam resolver o problema, ou amenizar a situação, como exempli- 
ficado pela resposta de um participante: "Podemos diminuir os efeitos com projetos de conscientização nas escolas, diminuir o acúmulo de lixo e poluição que começa na nossa própria casa”. As principais soluções mencionadas foram, então, as necessidades de preservação do ecossistema (16/32) e de reduzir a emissão de poluentes (13/32).

Houve 92 menções a possíveis responsáveis pela ocorrência das MCGs. Dentre essas, 42 adolescentes mencionaram a "ação humana", enquanto outros exatos 42 indicaram que a culpa dessa ocorrência seria "nossa". Optamos pela separação dessas duas categorias porque "nossa" envolve um caráter de autoimplicação mais efetivo, sendo a "ação humana" uma implicação de âmbito mais abstrato, não comprometida. Outros oito adolescentes (dos 92) afirmaram que os responsáveis são os governos e os interesses internacionais.

Outro resultado que deve ser mencionado foram as expressivas indicações de mensagens de conservação (139 indicações). Essa categoria se referia à presença de frases que versavam a respeito da necessidade de proteção do meio ambiente. Não eram recados focados no problema, não se relacionavam às MCGs, nem traziam ações concretas. Um exemplo desses recados é expresso pela frase de um dos respondentes: "Amigo, nós precisamos cuidar do ambiente, senão nós morreremos antes do tempo".

Ainda que as MCGs não estejam sendo diretamente retratadas nessas mensagens, elas não podem ser desconsideradas, afinal, refletem ideias frequentes nas percepções dos participantes (por seu número de indicações). Esse resultado pode sugerir uma preocupação com as questões ambientais desvinculada do conhecimento sobre as MCGs, ou, ainda, pode sugerir a presença de desejabilidade social (Kaiser et al., 2007), em que o respondente diz algo que espera ser a resposta socialmente correta ou desejada, e não o que realmente pensa ou faz.
Por fim, apenas 43 adolescentes mencionaram a importância de considerar a situação futura para si mesmo ou para todo o planeta diante dos impactos das MCGs. A ausência do futuro nas respostas dos participantes também surpreende, na medida em que essa dimensão temporal está diretamente associada à ocorrência das MCGs e a um tempo que virá na vida desses adolescentes.

Aspectos relativos ao consumo, às matrizes energéticas e ao uso do automóvel não apareceram nas respostas sobre MCGs desses adolescentes participantes. Essa ausência é relevante e sugere que tais temáticas podem representar caminhos a serem trilhados nas salas de aula ou em projetos de educação ambiental, pois, segundo a literatura, os adolescentes estão dispostos a discutir questões ambientais e a se engajar em ações de proteção (Wray-Lake et al., 2010).

\subsection{O engajamento dos adolescentes em ações de cuidado ambiental}

Dos 323 participantes, 174 (54\%) se declararam não cuidadores, ao afirmar que não praticam nem praticaram ações de cuidado ambiental. Ao mesmo tempo, 149 (46\%) se consideraram cuidadores, por afirmarem que se engajam, ou já se engajaram, em ações de cuidado.

Não houve associação estatisticamente significativa entre prática de cuidado ambiental e gênero, ou seja, as proporções de cada gênero entre os cuidadores não foram diferentes da proporção de cada gênero no total de participantes. Também não foram encontradas associações significativas entre prática de cuidado ambiental e as variáveis sociodemográficas número de irmãos, grau de instrução de pais e mães, idade e série escolar (nono ano, primeira e segunda séries). As séries escolares e as idades eram 
muito próximas, fato que pode ter contribuído para a ausência de associações nesses casos.

Todavia, foi encontrada associação significativa entre cuidado ambiental e o tipo de escola que os adolescentes frequentam $\left(\mathrm{x}^{2}=9,223 ; \mathrm{gl}=1 ; \mathrm{p} \leq\right.$ $0,002)$. Do total de participantes da escola pública ( $\mathrm{n}=142$ ), 52 (ou 37\%) se declararam cuidadores, enquanto, do total de participantes da escola particular $(\mathrm{n}=181)$, foram 97 (ou $54 \%$ ) os que se declararam cuidadores. Dito de outra maneira, enquanto na escola pública a maioria dos estudantes se declarou como não cuidadores (90/142, ou 63\%), na escola particular a maioria foi de cuidadores (97/181, ou 54\%).

Essa situação pode estar relacionada a diferenças institucionais quanto à educação ambiental, suas concepções, metodologias e práticas de ensino utilizadas pelos professores, a formação inicial e continuada destes, até a própria organização e funcionamento de cada tipo de escola (Fracalanza, 2004). Além disso, esses resultados podem ter várias outras interpretações. Entre elas, possíveis influências do contexto de vida do estudante de cada um dos tipos de escola (e.g., nível socioeconômico) no seu interesse pelas questões ambientais, nas condições disponíveis para o exercício de práticas pró-ambientais, ou até mesmo na percepção como praticante de alguma forma de cuidado ambiental.

\subsection{O que são ações de cuidado ambiental para os cuidadores?}

As ações de cuidado ambiental mencionadas foram categorizadas com base em estudos anteriores sobre comportamentos de proteção ecológica (Corral-Verdugo, 2001; Pinheiro \& Pinheiro, 2007) e, mais uma vez, as categorias não eram excludentes, pois um participante poderia mencionar uma ou mais ações. A tabela 2 sumariza e apresenta as ações de cuidado ambiental autorreportadas.

TABELA 2 - Frequência absoluta e percentuais de menção às ações de cuidado ambiental praticadas

\begin{tabular}{lccc}
\hline & \multicolumn{2}{c}{ Frequência de ocorrência } \\
\cline { 2 - 4 } Categoria/Ações praticadas & Absoluta & $\begin{array}{c}\text { Percentual por } \\
(n=211)\end{array}$ & $\begin{array}{c}\text { Cuidadores } \\
(n=149)\end{array}$ \\
\hline $\begin{array}{l}\text { Controle do lixo e estética } \\
\text { ambiental }\end{array}$ & 75 & $35,5 \%$ & $50 \%$ \\
$\begin{array}{l}\text { Plantação de mudas e } \\
\text { preservação do ecossistema }\end{array}$ & 39 & $18,5 \%$ & $26 \%$ \\
$\begin{array}{l}\text { Coleta seletiva de lixo e } \\
\text { reciclagem }\end{array}$ & 34 & $16 \%$ & $23 \%$ \\
$\begin{array}{l}\text { Campanhas de } \\
\text { conscientização ambiental e } \\
\text { movimentos }\end{array}$ & 31 & $15 \%$ & $21 \%$ \\
$\begin{array}{l}\text { Economia de água } \\
\text { Consumo responsável }\end{array}$ & 18 & $8,5 \%$ & $12 \%$ \\
$\begin{array}{l}\text { Economia de energia elétrica } \\
\begin{array}{l}\text { Diminuição do uso de } \\
\text { transporte privado }\end{array}\end{array}$ & 5 & $4 \%$ & $5 \%$ \\
\hline
\end{tabular}

Os resultados evidenciam uma amplitude considerada pequena de tipos de ações, apenas oito, em que os adolescentes se engajam. O estudo de Pinheiro e Pinheiro (2007), por exemplo, encontrou 14 categorias. Ainda assim, apenas controle do lixo e estética ambiental $(50 \% ; n=149)$, plantação de mudas e preservação dos ecossistemas $(26 \%)$, coleta seletiva do lixo e reciclagem (23\%) e campanhas de conscientização ambiental $(21 \%)$ foram mais expressivamente indicados.

Nessa classificação, a categoria controle do lixo e estética ambiental corresponde ao discurso relativo ao depósito adequado do lixo, para que não seja nas ruas, tornando-as sujas e desorganizadas. À guisa de exemplo, duas respostas mencionaram as seguintes práticas: "Tirar o lixo do chão e pôr no lixo, falar para as pessoas não jogarem lixo no chão" e "Jogar lixo nos lugares propícios à limpeza urbana”. 
Esse comportamento foi o mais lembrado pelos participantes, e isso pode ser reflexo de uma educação brasileira que discute, desde os primeiros anos de ensino, a necessidade de "jogar o lixo no lixo". Contudo, esse resultado ressalta a importância de debates sobre diversidade dos problemas humano-ambientais, destacando também outras ações de proteção que possam ser praticadas na realidade dos adolescentes.

Além disso, o efeito da desejabilidade social também pode estar presente (Kaiser et al., 2007), coerentemente com a percepção sobre MCGs já discutida aqui. Os participantes podem relatar ações de comportamento pró-ecológico simplesmente porque avaliam que isso é o que a sociedade espera como mais aceitável, mas não necessariamente é condizente com suas práticas.

Ainda assim, o lixo tem recebido considerável atenção, tendo sido mencionado como uma das principais causas para a ocorrência das MCGs. Na mesma direção, participar de ações de coleta seletiva do lixo e de reciclagem foi outra categoria expressivamente mencionada. Ela se referia a ações que auxiliassem essa coleta, tais como separar o lixo doméstico e o depósito em contentores adequados. Como exemplo, destacamos duas respostas dos adolescentes: “... busco facilitar a coleta de lixo, pela separação do lixo orgânico do inorgânico"; e "na minha casa há uma lixeira só para materiais que podem ser reciclados".

Essa categoria, somada com controle do lixo e estética ambiental, totaliza $73 \%$ das 149 respostas dos cuidadores. No entanto, este estudo optou por separar essas duas ações em categorias diferentes, tomando como base um caráter mais amplo da coleta seletiva, que contemplava respostas que iam além do esforço de despejar o lixo no local correto, incluindo ainda sua entrega para instituições responsáveis pela realização de reciclagem.
Neste estudo, a categoria de preservação de ecossistemas foi rotulada somando-se à plantação de mudas. Isso se deu porque todas as respostas (26\%) trouxeram a plantação de mudas como uma forma de preservar os ecossistemas, raramente mencionando outras maneiras, como a proteção de animais. Nesse sentido, uma resposta retrata o seguinte: "Na minha antiga escola tinha um projeto que (sic) fiz parte para preservar o meio ambiente e plantávamos árvores". Importa destacar que, assim como o controle do lixo, a plantação de sementes e mudas é um assunto bastante discutido e estimulado pela educação formal.

Nessa direção, as participações em campanhas de conscientização e movimentos foram outra categoria com alguma expressão (21\%). Geralmente, tais ações são realizadas com vínculo a instituições, como palestras nas escolas, ou projetos realizados por estas e, ainda, por igrejas ou conselhos comunitários, no que se refere ao contexto dos participantes deste estudo.

Outros resultados que também chamam a atenção são as baixas indicações de ações relativas à economia de água (12\%) e energia (3\%). Esses dois pontos são relevantes para a temática das MCGs, e as poucas indicações sugerem coerência com o desconhecimento do assunto ou fraca consideração do problema. Além disso, a mídia brasileira, assim como as escolas, costuma discutir e estimular essas ações, principalmente diante de crises hídricas oriundas de períodos de seca. Todavia, muitas vezes essas ações são associadas a aspectos financeiros. Por isso, uma possível interpretação para essas baixas indicações reside no fato de que os adolescentes se lembram dessas ações como questão econômica, e não como questão de cuidado ambiental. Essa interpretação tem base em outros estudos, que estabeleceram que quem conserva energia geralmente $o$ faz por razões desconectadas do ambiente, tais como economizar na conta de luz (Whitmarsh, 2009). 
Além dessas ações, o consumo responsável ou consciente foi pouco mencionado (5\%). Essa categoria se referia às ações para diminuição do consumo ou aquisição de atitudes reflexivas sobre o que se está comprando, escolha de produtos e seus impactos ambientais. A diminuição do uso de transporte privado, por sua vez, foi uma ação mencionada por apenas um adolescente. Essa realidade também merece atenção: ela evidencia uma pequena variabilidade na percepção sobre o que pode ser feito pelos adolescentes, principalmente nas práticas mais diretamente efetivas no combate às MCGs.

Em síntese, todas as ações adotadas pelos adolescentes são relevantes. No entanto, os resultados mostram uma pequena diversidade destas e desconhecimento sobre os aspectos mais relacionados às mudanças climáticas. Por isso, ações de comunicação sobre o problema podem explorar mais esses aspectos e também os impactos para a realidade local brasileira, para além do derretimento das geleiras - consequência mais mencionada. É interessante que se reflita, ainda, sobre o papel da família nessas ações, e estudos futuros sobre comportamento pró-ecológico podem levar esse aspecto em conta, afinal, os adolescentes podem possuir graus diferentes de independência para se engajar em uma ou mais práticas, e influências parentais podem tomar parte nesse processo (Ojala, 2013).

\subsection{Associações entre práticas de cuidado ambiental e a percepção das MCGs}

O objetivo central deste estudo foi investigar se os adolescentes cuidadores iriam perceber de maneira diferenciada as mudanças climáticas.

Os resultados indicaram associações significativas entre a percepção das causas das MCGs e a adoção de práticas de cuidado relativas ao controle do lixo $\left(\mathrm{x}^{2}=3,257 ; \mathrm{gl}=1 ; \mathrm{p} \leq 0,05\right)$, em que os participantes que se engajam nesse tipo de ação mencionaram mais as causas do problema (63\%) do que os que não se engajam (51\%). Tendência similar foi observada entre perceber as causas do problema e se engajar nas ações referentes a coleta seletiva e reciclagem $\left(72 \%>52 \% ; \mathrm{x}^{2}=4,430 ; \mathrm{gl}=1 ; \mathrm{p} \leq\right.$ $0,05)$. Esses achados coincidem com os resultados de Whitmarsh (2009), ao mencionar que as pessoas que agem se preocupando com as MCGs se engajam em atividades diversas, para além da conservação de energia, e que a reciclagem seria uma das atividades mais populares para esse grupo, o que corrobora a ideia de que o cuidado ambiental é pluridimensional (Gurgel \& Pinheiro, 2011).

Por outro lado, é importante lembrar que, para os adolescentes, a causa mais expressiva das MCGs foi a poluição por acúmulo de lixo. Sendo assim, é coerente que as pessoas se engajem nessas ações como forma de combate. Ainda, convém ressaltar que a questão do lixo está ao alcance do indivíduo, enquanto outros problemas e soluções podem ser percebidos como amplos demais para o nível individual. Essa possível interpretação reforça a necessidade de comunicação sobre as MCGs pelas escolas e pela mídia, ressaltando outras ações de mitigação relacionadas ao problema que possam ser praticadas levando-se em conta as condições de existência dos adolescentes. Assim, estimulam-se comportamentos outros, além do controle do lixo, principalmente se este se reduzir à estética ambiental.

Não houve associações significativas entre consequências das MCGs, possíveis soluções do problema e o cuidado ambiental. A falta de associação entre esses aspectos pode indicar, mais uma vez, que as MCGs não são percebidas como um problema ambiental comum, visível, concreto (Pawlik, 1991; Uzzel, 2000; Weber \& Stern, 2011). Assim, seus impactos e soluções talvez devam ser 
tratados ao nível local, considerando as respectivas escalas espacial e temporal, de acordo com as quais os adolescentes podem realizar ações concretas em seu cotidiano.

Sundblad et al. (2009) e Weber \& Stern (2011) mencionam o pouco conhecimento que existe sobre as consequências das MCGs, além dos variados graus de incertezas que as cercam. Nesse sentido, a falta de conhecimento pode contribuir para a falta de associações nesta investigação. O conhecimento é um elemento necessário para se engajar em ações de mitigação, e os estudos vêm evidenciando consistentemente a grande confusão conceitual que cerca o tema (Bord et al., 1998; Dunlap, 1998; Oppenheimer \& Todorov, 2006; Sundblad et al., 2009). "Não saber" o que fazer a respeito dos impactos das MCGs é, portanto, uma barreira para mitigação (Gifford, 2011).

Sublinhem-se, ainda, as associações significativas entre quem mencionou atribuição de responsável pelas MCGs e quem se engajou em: plantação de mudas e preservação de ecossistemas $(44 \%>$ $\left.26 \% ; \mathrm{x}^{2}=4,969 ; \mathrm{gl}=1 ; \mathrm{p} \leq 0,05\right)$, economia de energia elétrica $\left(80 \%>28 \% ; \mathrm{x}^{2}=6,617 ; \mathrm{gl}=1 ; \mathrm{p}\right.$ $\leq 0,05)$ e participação em campanhas de conscientização $\left(48 \%>26 \% ; x^{2}=6,669 ; \mathrm{gl}=1 ; \mathrm{p} \leq 0,05\right)$, indicando que os adolescentes que se engajaram nessas ações mencionaram mais os responsáveis pelo problema do que os que não se engajaram. Esses resultados sugerem uma estreita relação entre atribuição de responsabilidade e cuidado ambiental, o que é coerente com achados de outros autores, ao apontarem o senso de responsabilidade como preditor do comportamento pró-ecológico (Kaiser \& Shimoda, 1999).

Ainda assim, há evidências de sinal contrário que importa assinalar: a atribuição de responsáveis foi associada com controle do lixo e estética ambiental - no entanto, em tendência oposta $\left(\mathrm{x}^{2}\right.$
$=4,621 ; \mathrm{gl}=1 ; \mathrm{p} \leq 0,05)$. Os participantes que se engajaram nessas ações apontaram menos responsáveis pelo problema (19\%) do que os que não se engajaram nessa forma de cuidado ambiental (32\%). Novamente, o efeito da desejabilidade social pode explicar essa tendência (Kaiser et al., 2007). De fato, na realidade norte-rio-grandense, quiçá brasileira, se banalizou o já mencionado discurso "não jogue o lixo no chão, jogue o lixo no lixo", sendo possível a reprodução desse slogan sem se pensar muito em seu significado, sem considerar problemas ambientais, ou até sem agir em consonância.

Plantar mudas e sementes também é um discurso comumente reproduzido, mas, diferentemente, costuma tornar-se ação concreta para além do discurso, sendo frequente a vinculação a projetos em escolas e instituições, como a criação de hortas comuns, por exemplo. Por outro lado, a deposição do lixo em locais próprios costuma ser de ordem privada e pode mais facilmente ficar apenas no discurso. Diante disso, novas investigações baseadas na observação do comportamento pró-ecológico podem ser conduzidas, a fim de entender essa realidade de maneira mais aprofundada.

Por fim, este estudo encontrou associação significativa entre as mensagens de conservação e a coleta seletiva e a reciclagem $\left(\mathrm{x}^{2}=4,253\right.$; $\mathrm{gl}=1 ; \mathrm{p} \leq 0,05)$. Os adolescentes que se engajaram nesse tipo de comportamento mencionaram menos mensagens de conservação $(26 \%)$ do que os que não se engajaram (45\%). As mensagens de conservação refletiam um sentido de desejabilidade social, afirmando a necessidade de proteger genericamente o meio ambiente, sem mencionar como e sem relacionar-se com as MCGs. Por outro lado, separar materiais e enviá-los para a reciclagem, como já mencionado, são ações mais ativas e mais amplas conceitualmente do que o controle do lixo com fins estéticos, além de serem ações associadas 
à consideração pelas MCGs em outros estudos (Whitmarsh, 2009). Sendo assim, é coerente o resultado de que quem pratica ações relacionadas a coleta seletiva e reciclagem menciona menos a importância de cuidar do meio sem oferecer formas concretas para isso.

\section{Considerações finais}

Em síntese, uma percepção confusa e uma falta de conhecimento claro sobre mudanças climáticas citadas por outros autores (Dunlap, 1998; Cabecinhas et al., 2006; Oppenheimer \& Todorov, 2006; Sundblad et al., 2009; Swim et al., 2009; Stern, 2011; Clayton et al., 2015) também foram encontradas nesta investigação. A confusão conceitual residiu na dificuldade em distinguir causas mais diretas do problema e a conexão dele com outros problemas ambientais. As interações entre vários elementos da dinâmica da mudança no clima são desconhecidas pelo público em geral (Weber \& Stern, 2011). Apesar disso, este estudo encontrou maior indicação de causas do que de consequências, ainda que tenham sido mais associadas a problemas ambientais locais, mais concretos, como o lixo.

Já em relação ao conceito de cuidado ambiental, os achados corroboraram a literatura da área, quando se afirma que a noção de cuidado ambiental é bem compreendida pelas pessoas (Pinheiro \& Pinheiro, 2007). Durante a aplicação do instrumento, não foram observadas dúvidas relativas a esse item, e a estratégia de descrição aberta da ação se tornou ideal por permitir que o sentido dado ao cuidado ambiental fosse expresso pelos adolescentes, validando suas respostas.

Por meio desses resultados, é possível sugerir que os projetos visando a educação ambiental busquem aumentar o escopo de ações realizáveis pelos jovens, tornando outros comportamentos tão populares quanto os relacionados ao lixo e enfatizando as relações de práticas locais com problemas globais (Clayton et al., 2015; Devine-Wright et al., 2015). A discussão sobre as arenas multifacetadas em que entram em conflito os interesses internacionais, empresariais, as vulnerabilidades e interesses sociais e aspectos relativos à saúde humana também corresponde a um caminho a ser trilhado ao informar sobre MCGs. Tais aspectos foram inexpressivamente mencionados nas respostas dos adolescentes.

Diante desse cenário, responder efetivamente à ameaça da mudança no clima requer o desenvolvimento de um corpo acadêmico de pesquisas, que some todos os relevantes processos humanos e psicológicos envolvidos na temática (Devine-Wright, 2013). Nesse sentido, esta investigação lança luz à maneira como os adolescentes percebem o problema e como se comportam para proteção ecológica e ressalta aspectos que continuam obscuros para a tradução desse problema em ações locais.

$\mathrm{O}$ estudo apresenta algumas limitações que importa destacar. O fato de se basear em uma estratégia de autorrelato abre espaço para o efeito da desejabilidade social nas respostas. No intuito de minimizar esses efeitos, só foram aceitas como afirmativas as respostas que continham as caracterizações das ações de cuidado. Além disso, ao adotar o caráter imaginativo na questão sobre MCGs, buscou-se diminuir um eventual caráter de prova/exame existente nas escolas como avaliação de conteúdo.

Reconhece-se, ainda, que o estudo lidou com uma população de poucos adolescentes norte-rio-grandenses, estudantes na cidade de Natal. Pesquisas futuras com um maior número de participantes oriundos de geografias variadas poderão prover indicadores mais sólidos e generalizáveis. Apesar disso, esta investigação proporcionou uma primeira lente para essa realidade, associando as ações locais 
de proteção ambiental às concepções de MCGs, e, com isso, sugere possibilidades para o trabalho com educação ambiental com jovens das mais diversas realidades.

Entre essas possibilidades, informar a respeito de medidas de mitigação, tais como a redução de carbono, por meio da promoção e recuperação de áreas que foram degradadas para pastagem; a substituição dos combustíveis fósseis usados pela indústria, com a geração de energia solar e eólica; a implementação de sistemas de transporte público integrados e mais eficientes, além da redução de veículos automotivos nas áreas urbanas e do necessário freio ao desmatamento na Amazônia (PBMC, 2014), é um assunto que pode ser debatido com os adolescentes e jovens (Wray-Lake et al., 2010) e que escapou ao discurso deles nesta investigação. $\mathrm{O}$ assunto faz parte das grades curriculares das escolas pesquisadas. No entanto, resta a dúvida sobre se este tem sido de fato abordado, ou qual o contexto dessa abordagem, já que estão ausentes do discurso do adolescente. Pesquisas futuras podem centrar-se também nessas realidades institucionais.

As ações de mitigação citadas podem ser do âmbito da gestão pública e privada, mas outras ações individuais e coletivas também são demandadas. Desde o trabalho comunitário para cobrança dessas

\section{Referências}

Bardin, L. Análise de conteúdo. Lisboa: Edições 70, 1977. Barros, H. C. L.; Pinheiro, J. Q. Dimensões psicológicas do aquecimento global conforme a visão de adolescentes brasileiros. Estudos de Psicologia, 18(2), 173-182, 2013. Disponível em: <www.scielo.br/epsic>.

Bauer, M. W. Análise de conteúdo clássica: uma revisão. In: Bauer, M. W.; Gaskell, G. (Orgs.). Pesquisa qualitativa com texto, imagem e som. Petrópolis: Vozes, 2002. p. 189-217. medidas, aceitação delas quando implantadas e mudanças nos padrões de transporte e de consumo energético, até a promoção de plantio e manutenção de áreas verdes, realização de um consumo consciente, reutilização e engajamento em reciclagem. Todas essas medidas contribuem direta ou indiretamente para reduzir a emissão de gases de efeito estufa na atmosfera, mitigam as mudanças climáticas globais (PBMC, 2014) e podem ser objeto de informação, debate e reflexão com os adolescentes.

Portanto, nesse cenário de problemas globais, estudos que objetivem compreender a relação entre aspectos psicológicos, comportamentais e sociais a partir das localidades se tornam relevantes. Podem contribuir para promover estilos de vida sustentáveis e o chamado afeto planetário, um comprometimento com o ambiente que vai além das localidades e das diferentes gerações.

\section{Agradecimentos}

Os autores agradecem o Conselho Nacional de Desenvolvimento Científico e Tecnológico (CNPq) pelo apoio financeiro recebido, na forma de bolsa de mestrado para a autora e bolsa de produtividade em pesquisa para o autor.

Boff, L. Saber cuidar: ética do humano - compaixão pela Terra. Petrópolis: Vozes, 1999.

Bord, R. J.; Fisher, A.; O’Connor, R. E. Public perceptions of global warming: United States and international perspectives. Climate Research, 11, 75-84, 1998. doi: 10.12691/ ajap-1-3-6

Cabecinhas, R.; Lázaro, A.; Carvalho, A. Lay representations on climate change. Proceedings of IAMCR's 
25th Conference, p. 504-508, Cairo: IAMCR, 2006. Disponível em: $<$ http://repositorium.sdum.uminho.pt/bitstream/1822/5335/1/Cabecinhas_Lazaro_Carvalho_IAMCR_2006.pdf $>$.

Clayton, S.; Devine-Wright, P.; Stern, P.; Whitmarsh, L.; Carrico, A.; Steg, L.; Bonnes, M. Psychological research and global climate change. Nature Climate Change, 5, 640-646, 2015. doi: $10.1038 /$ nclimate 2622

Corner, A. Science literacy and climate views. Nature climate change, 2, 710-711, 2012. doi: 10.1038/nclimate1700.

Corral-Verdugo, V. Comportamiento proambiental. Santa Cruz de Tenerife: Editorial Resma, 2001.

Corral-Verdugo, V. Psicologia de la Sustentabilidad: un análisis de lo que nos hace pro-ecológicos y pro-sociales. Cidade do México: Trillas, 2010.

Devine-Wright, P. Think global, act local? The relevance of place attachments and place identities in a climate changed world. Global Environmental Change, 23, 61-69, 2013. doi: 10.1016/j.gloenvcha.2012.08.003

Devine-Wright, P.; Price, J.; Leviston, Z. My country or my planet? Exploring the influence of multiple place attachments and ideological beliefs upon climate change attitudes and opinions. Global Environmental Change, 30, 68-79, 2015. doi: 10.1016/j.gloenvcha.2014.10.012

Diniz, R. F.; Pinheiro, J. Q. Cuidado ambiental em tempos de sustentabilidade: relação entre compromisso pró-ecológico e orientação de futuro. Psico, 45(3), 387-394, 2014. doi: 10.15448/1980-8623.2014.3.17321

Dunlap, R. E. Lay perceptions of global risk - public views of global warming in cross-national context. International Sociology, 13(4), 473-498, 1998. doi: $10.1177 / 026858098013004004$

Fracalanza, H. As pesquisas sobre educação ambiental no Brasil e as escolas: alguns comentários preliminares. In: Taglieber, J. E.; Guerra, A. F. S. (Orgs.). Pesquisa em Educação Ambiental: pensamentos e reflexões de pesquisadores em Educação Ambiental. Pelotas: Universitária/ UFPel, 2004. p. 55-77.

Gifford, R. Psychology's essential role in alleviating the impacts of climate change. Canadian Psychology, 49(4), 273-280, 2008. doi: 10.1037/a0013234.
Gifford, R. The dragons of inaction: Psychological barriers that limit climate change mitigation and adaptation. American Psychologist, 66(4), 290-302, 2011. doi: 10.1037/ a0023566.

Günther, H. Como elaborar um questionário. In: Pinheiro, J. Q.; Günther, H. (Orgs.). Métodos de pesquisa nos estudos pessoa-ambiente. São Paulo: Casa do Psicólogo, 2008. p. 105-147.

Günther, H.; Elali, G. A.; Pinheiro, J. Q. A abordagem multimétodos em estudos pessoa-ambiente: características, definições e implicações. In: Pinheiro, J. Q.; Günther, H. (Orgs.). Métodos de pesquisa nos estudos pessoa-ambiente. São Paulo: Casa do Psicólogo, 2008. p. 369-396.

Gurgel, F.; Pinheiro, J. Q. Compromisso pró-ecológico. In: Cavalcante, S.; Elali, G. (Orgs.). Temas Básicos em Psicologia Ambiental. Petrópolis: Vozes, 2011. p. 267-280.

Hart, A. R. Children's Participation. The theory and practice of involving the young citizens in community development and environmental care. New York: UNICEF, 1997.

Heft, H.; Chawla, L. Children as agents in sustainable development: the ecology of competence. In: Spencer, C.; Blades, M. (Orgs.), Children and their environments: learning, using and designing spaces. Cambridge, Reino Unido: Cambridge University Press, 2005. p. 199-216.

IPCC - Intergovernmental Panel on Climate Change. Climate Change 2014: Synthesis Report. Contribution of Working Groups I, II and III to the Fifth Assessment Report of the Intergovernmental Panel on Climate Change [Core Writing Team, R.K. Pachauri and L.A. Meyer (eds.)]. IPCC, Geneva, Switzerland, 151 p., 2014. Disponível em: $<$ http:// ipcc.ch/report/ar5/syr/>. Acesso em: mar. 2016.

Kaiser, F. A general measure of ecological behavior. Journal of Applied Social Psychology, 28, 395-442, 1998. doi: 10.1111/j.1559-1816.1998.tb01712.x

Kaiser, F.; Shimoda, T. Responsibility as a predictor of ecological behavior. Journal of Environmental Psychology, 19(3), 243-25, 1999, doi: 10.1006/jevp.1998.9123

Kaiser, F.; Oerke, B.; Bogner, F. Behavior-based environmental attitude: Development of an instrument for adolescents. Journal of Environmental Psychology, 27, 242-251, 2007. doi: $10.1016 /$ j.jenvp.2007.06.004 
Kuhnen, A.; Higuchi, M. I. G. Percepção Ambiental. In: Cavalcante, S.; Elali, G. (Orgs.). Temas básicos em Psicologia Ambiental. Petrópolis: Vozes, 2011. p. 250-266.

McDonald, R.I.; Chai, H. Y; Newell, B. R. Personal experience and the 'psychological distance' of climate change: An integrative review. Journal of Environmental Psychology, 44, 109-118, 2015. doi: 10.1016/j.jenvp.2015.10.003

Moser, G.; Uzzel, D. Psychology and the challenge of global environmental change. IHDP UPDATE [Newsletter of the International Human Dimensions Programme on Global Environmental Change], 4, 1-2, 2004. Disponível em: $<$ http://ihdp.unu.edu/ $>$.

Ojala, M. Coping with Climate Change among adolescents: implications for subjective well-being and environmental engagement. Sustainability, 5, 2191-2209, 2013. doi: $10.3390 /$ su5052191

O' Neill, S.; Nicholson-Cole, S. "Fear won't do it": promoting positive engagement with climate change through visual and iconic representations. Science Communication, 30, 355- 379, 2009. doi: 10.1177/1075547008329201

Oppenheimer, M.; Todorov, A. Global Warming: the psychology of long term risk [Guest Editorial]. Climate Change, 77, 1-6, 2006. doi: 10.1007/s10584-006-9086-6.

Pato, C. M. L.; Campos, C. B. Comportamento ecológico. In: Cavalcante, S.; Elali, G. (Orgs.). Temas básicos em Psicologia Ambiental. Petrópolis: Vozes, 2011. p. 122-143.

Pawlik, K. The psychology of global environmental change: some basic data and an agenda for cooperative international research. International Journal of Psychology, 26(5), 547563, 1991. doi: 10.1080/00207599108247143

Pawlik, K. Global Change as heuristic challenge to Psychology. IHDP UPDATE [Newsletter of the International Human Dimensions Programme on Global Environmental Change], 4, 3, 2004. Disponível em: <http://ihdp.unu.edu/>.

PBMC - Painel Brasileiro de Mudanças Climáticas. Base científica das mudanças climáticas. Contribuição do Grupo de Trabalho 1 do Painel Brasileiro de Mudanças Climáticas ao Primeiro Relatório da Avaliação Nacional sobre Mudanças Climáticas [T. Ambrizzi e M. Araújo, (Orgs.)]. Rio de Janeiro: COPPE, Universidade Federal do Rio de Janeiro, 2014. Disponível em: <http://www.pbmc.coppe. ufrj.br/documentos/RAN1_completo_vol1.pdf.>. Acesso em: fev. 2016.
Pinheiro, J. Q.; Pinheiro, T. F. Cuidado ambiental: Uma ponte entre Psicologia e cuidado ambiental? Psico, 38(1), 25-34, 2007. Disponível em: <www.revistaseletronicas.pucrs.br>.

Raymundo, L. S.; Kuhnen, A. A psicologia e a educação ambiental. Revista de Ciências Humanas, 44(2), p. 435-450, 2010. doi: 10.5007/2178-4582.2010v44n2p435

Renaud-Dubé, A.; Taylor, G.; Lekes, N.; Koestner, R.; Guay, F. Adolescents' motivation toward the environment: age-related trends and correlates. Canadian Journal of Behavioural Science, 42 (3), 194-199, 2010. doi: 10.1037/ a0018596

Sommer, B.; Sommer, R. A practical guide to behavior research: tools and techniques. Nova York: Oxford University Press, 1997.

Stern, P. C. Contributions of Psychology to limiting climate change. American Psychologist, 66(4), 303-314, 2011. doi 10.1037/a0023235

Stern, P.C.; Young, O.R.; Druckman, D. Global environmental change: understanding the human dimensions. Washington, D.C: National Academy of Sciences, 1992. Disponível em: <http://www.nap.edu/catalog/1792.html > .

Sundblad, E.; Biel, A.; Gärling, T. Knowledge and confidence in knowledge about climate change among experts, journalists, politicians, and laypersons. Environmental and Behavior, 41, 281-302, 2009. doi: 10.1177/0013916508314998

Swim, J.; Clayton, S.; Doherty, T.; Gifford, R.; Howard, G.; Reser, J.; Stern, P.; Weber, E. Psychology and global climate change: addressing a multi-faceted phenomenon and set of challenges. [Report by the American Psychological Association's Task Force on the interface between Psychology and global climate change]. Washington, DC: American Psychological Association, 2009. Disponível em: <http:// www.apa.org/releases/climate-change.pdf $>$.

Tabachnick, B. G.; Fidell, L. S. Using multivariate statistics. Nova York: Harper Collins, 1996.

Uzzell, D. L. The Psycho-Spatial Dimension to Global Environmental Problems. Journal of Environmental Psychology, 20(4), 307-318, 2000. doi: 10.1006/jevp.2000.0175

Weber, E. U.; Stern, P. C. Public understanding of climate change in the United States. American Psychologist, 66(4), 315-328, 2011. doi: 10.1037/a0023253 
Whitmarsh, L. Behavioural responses to climate change: Asymmetry of intentions and impacts. Journal of Environmental Psychology, 29, 13-23, 2009. doi: 10.1016/j. jenvp.2008.05.003

Winkel, G.; Saegert, S.; Evans, G. W. An ecological perspective on theory, methods, and analysis in environmental psychology: advances and challenges. Journal of Envi- ronmental Psychology, 29, 318-328, 2009. doi: 10.1016/j. jenvp.2009.02.005

Wray-Lake, L.; Flanagan, C.; Osgood, D.W. Examining trends in adolescent environmental attitudes, beliefs, and behaviors across three decades. Environment and Behavior, 42(1), 61-85, 2010. doi: 10.1177/0013916509335163 